\title{
Application of the Craig-Bampton method to blade rotation
}

\author{
Jose Leoro ${ }^{1,}{ }^{*}$, Vladislav Borisenko ${ }^{1}$, Egor Degilevich $^{1}$, and Anton Didenko ${ }^{1}$ \\ ${ }^{1}$ Peter the Great St. Petersburg Polytechnic University (SPbPU), 195251, St. Petersburg, Russia
}

\begin{abstract}
The Craig-Bampton method has repeatedly shown its effectiveness in modelling the deformable solid body motion and in practical experience of implementing this approach in engineering software. It reduces the motion equations system which is obtained by approximating small elastic displacements of the body finite element model with a set of acceptable modes. The application of this method in engineering improves the computing time performance and requires less computational resources. In this article the effectiveness of using the Craig-Bampton method for the blade rotation is evaluated. The need to consider the inertial relationship between large body displacements and elastic deformations was investigated. As a result of the numerical solution, it was obtained that the method works correctly for the investigated task. It allows us to conclude that the Craig-Bampton method is applicable for the blade rotation process.
\end{abstract}

\section{Introduction}

The Craig-Bampton method is used in the analysis of diverse objects, whether it is a moving or a static construction, that is either under the influence of forces changing in time or interacting with other objects.

There are many applied problems when the maximum calculations accuracy is required, and it is important to have high reliability in the machinery operations and in other various constructions. One of the latest researches [1] showed the significant reduction of computing time in vibroacoustic analysis of SSTL300 spacecraft with a complex structure using the Craig-Bampton method. As a result of comparisons, it was found that the accuracy of the method for DOF in physical and modal coordinate systems was almost identical. This fact indicates a good reliability of the technique which makes it possible to store the complete data set of the vibroacoustic solution in a very compact form. It should be noted that the model may consist of different types of elements and the modelling itself is often carried out by several teams, which may lead to incompatibility of formats. However, the CraigBampton method for subsystems makes it is possible to obtain reduced stiffness and mass matrices in a universal format.

The method has also found its application in the engines design as reported, for instance, in the study of a marine vessel four-stroke diesel engine [2]. In addition, the Craig-Bampton method can be extremely helpful in studying the vibrations of considerable length objects.

\footnotetext{
*Corresponding author: zhilenkovanton@gmail.com
} 
As an example, the length of a drill string can reach several kilometres [3]. Oil production requires high reliability of drilling operations, so computer models can be cumbersome. It requires a lot of memory and computational time to consider various kinds of non-linear effects. However, the Craig-Bampton method helps us to reduce the number of DOF. As mentioned in the previous studies, the method dramatically reduces the calculation time, keeping the relative error about several percent. Another important advantage of the method is that the transmission of information about subsystems in the form of reduced matrices allows design organizations to keep commercial secrets without disclosing unnecessary information.

However, no one has focused on the possibility of using the Craig-Bampton method for rotating blades yet. And that is the main theme of this work.

\section{Modal superposition}

In the Craig-Bampton method, the deformable body system of motion equations is reduced with the usage of FEM. It is implemented by approximating small elastic body displacements with a set of acceptable forms. Before focusing on the mathematical interpretation, it is necessary to analyse the point of the modal superposition, which lies at the basis of CraigBampton method.

We consider only small linear deformations of the body relative to the local coordinate system (hereinafter LCS). The LSCs themselves are affected by a large nonlinear motion in the global coordinate system (hereinafter GCS).

Modal superposition consists in the representation of complex modes as a linear combination of simple ones. The coordinates of all FEM nodes $\mathrm{x}_{n}$ in the LSC are written in the following form [4]:

$$
\mathrm{x}_{n}=\sum_{i=1}^{M} h_{i} \mathrm{w}_{i},
$$

where $M$ is the total number of used modes, $h$ is the set of forms, $w$ is the set of modal coordinates, and the index $i=\overline{1, M}$ is responsible for the mode number. Equation (1) can be also written in matrix form:

$$
\mathrm{x}_{n}=\mathrm{Hw},
$$

where $\mathrm{H}$ is a complete modal matrix describing the complete set of modes used and representing the transformation of a small set of modal coordinates $\mathrm{w}$ into a large set of physical coordinates $\mathrm{x}_{n}$.

The modal superposition allows us to find the deformation components of the blade, and it is the basis of the Craig-Bampton approximation method.

\section{Content of the Craig-Bampton method}

The Craig-Bampton method allows selecting the eigenforms of vibrations (modes) so that the maximum amount of deformations can be captured with a minimum amount of modal coordinates. In order to achieve this, the method involves selecting a subset of nodes that are not subject to modal superposition. Such nodes are called boundary (interface) nodes. They are affected by force components and joints, and that is why an approximate motion representation of such nodes is undesirable. The division of the entire set of nodes into 
boundary and interior nodes is a preparatory step in the Craig-Bampton algorithm. The next two steps are:

1) the static modes (bounding modes) are calculated by giving each boundary node a unit displacement in all degrees of freedom, while all other boundary nodes are fixed;

2) calculation of the vibrations eigenforms is performed by fixing the boundary nodes.

The main result of the Craig-Bampton method and its final stage is the construction of a modal matrix.

The coordinates of all the boundary nodes of the FEM are denoted as $\mathrm{x}_{n F}$, and the coordinates of the internal nodes as $\mathrm{x}_{n R}$, then equation (2) can be overwritten as follows:

$$
\mathrm{x}_{n}=\left\{\begin{array}{l}
\mathrm{x}_{n F} \\
\mathrm{x}_{n R}
\end{array}\right\}=\left[\begin{array}{cc}
\mathrm{I} & 0 \\
\mathrm{H}_{I C} & \mathrm{H}_{I N}
\end{array}\right]\left\{\begin{array}{l}
\mathrm{w}_{C} \\
\mathrm{w}_{N}
\end{array}\right\}
$$

where $\mathrm{I}$ is the unit matrix, 0 is the zero matrix, $\mathrm{H}_{I C}$ is the modal matrix describing the physical displacement of internal DOF in the restricting modes, $\mathrm{H}_{I N}$ is the physical displacement of internal DOF eigenforms, $\mathrm{w}_{C}$ means modal coordinates of the restricting modes, and $\mathrm{w}_{N}$ means modal coordinates of the fixed eigenforms.

The construction of modal matrices is impossible without determining the generalized matrices of mass $\bar{M}$ and stiffness $\bar{C}$, which are related by the following relations [4]:

$$
\begin{aligned}
\overline{\mathrm{M}} & =\mathrm{H}^{T} \mathrm{M}_{F E M} \mathrm{H}, \\
\overline{\mathrm{C}} & =\mathrm{H}^{T} \mathrm{C}_{F E M} \mathrm{H},
\end{aligned}
$$

where $\mathrm{M}_{F E M}$ and $\mathrm{C}_{F E M}$ are the complete mass and stiffness matrices, respectively.

\section{General equations of elastic body motion based on the Craig- Bampton method}

As noted earlier, the Craig-Bampton method allows deriving the equations of elastic body dynamics for problems where elastic bodies are subject to small deformations, but at the same time participate in a large displacement as part of a mechanical system. Therefore, it is practically significant to derive general equations of motion for elastic bodies from the Lagrange [4] equation using the theory presented in equation (2).

It is well known that the basic equations of the elastic body dynamics are derived from the Lagrange equation of the second kind:

$$
\frac{d}{d t}\left(\frac{\partial T}{\partial \dot{q}}\right)-\frac{\partial T}{\partial q}+\frac{\partial U}{\partial q}=s
$$

where $T$ is the kinetic energy, $U$ is the potential energy, $\mathrm{q}$ is the vector of generalized coordinates of the body, $s$ is the vector of generalized forces. Then all the terms of equation (5) are to be gradually find. Vector q is choosen as a set of two vectors: the six-dimensional vector $(r, \varphi)$, which characterizes the position of the LCS relative to the GCS, and the vector of modal coordinates $\mathrm{w}$ :

$$
\mathrm{q}=(\mathrm{r}, \varphi, \mathrm{w})
$$

Vectors $r$ and $\varphi$ can be obtained as:

$$
\dot{\mathrm{r}}=\mathrm{v}, \dot{\varphi}=\omega,
$$

where $\mathrm{v}$ and $\omega$ are the linear and angular velocities of the local reference frame relative to the global reference frame. 
If FEM of a body consists from $N$ nodes, then the number of DOF in the investigated model is $6 N$ and the size of the complete modal matrix is $6 N \times M(M$ is the number of modes used). The equation for calculating the kinetic energy has the following form:

$$
T=0.5 \cdot \dot{\mathrm{q}}^{T} \mathrm{M} \dot{\mathrm{q}}
$$

where $\mathrm{M}$ is the generalized mass matrix.

After introducing additional vectors of generalized inertia forces $\mathrm{k}$, generalized elastic forces $\mathrm{s}_{c}$, damping forces $\mathrm{s}_{D}$, gravity $\mathrm{s}_{g}$, and active concentrated forces $\mathrm{s}_{a}$ the following expression is obtained:

$$
M \ddot{q}=s_{g}+s_{a}+s_{D}+s_{c}+k+R,
$$

where $\mathrm{R}$ is the bond reaction force.

Equation (9) is a general equation of the solid dynamics based on the Craig-Bampton method and has the same form as the generalized equation of solid motion [4]. Equation (9) has a great practical importance, since it helps to model bodies with small linear deformations which experience a large nonlinear global motion.

In relation to the problem under consideration, the most general equations are obtained. This is due to the fact that no additional approximations are introduced. This fact is an advantage of the problem, since there is an opportunity not to distort the dynamic behavior of the body, to which the approximation can lead due to the addition of an error. Besides, such an equation allows not only investigating the diagonal mass matrix of the body, which is usually obtained from the FEM package, but also exploring its consistent form, which simplifies computational operations.

The studies performed on the practical application of the Craig-Bampton method focused on the difficulties in using the general equations of motion [4]. The most significant difficulties we found to consist in the presence of zero mass-inertia characteristics by some DOF and the following incorrect behavior description of the entire body.

The first problem is typical in the usage of three-dimensional elements (3D modeling) and is caused by the lack of masses of three-dimensional elements by rotational DOF in most cases. It means that direct use of the described algorithm is impossible since the generalized mass matrix is not positively defined.

The second problem can also occur with $3 \mathrm{D}$ modeling, although it is common in a number of other cases. In 3D modeling the inertia tensor is calculated from the distribution of translational masses and does not take into account local rotational movements. Therefore, if the model is not highly divided into finite elements, the error value will be quite large.

In order to eliminate such problems, it is necessary to modify the general equations of elastic bodies motion derived on the basis of the Craig-Bampton method with the help of the approximation.

\section{Methods for simulating blades}

Various methods are used to model the blades. Each has its own advantages and disadvantages in different kinds of aspects. But always the choice between the blade study models depends entirely on the task at hand. In [5] the authors reviewed various main rotor blade modeling techniques and described the strengths and weaknesses of each approach. It is noted that coning, longitudinal flapping, and lateral flapping angles can be used to describe the movement of the blades in the case of steady flight. The approach is called the rotor plane method. It allows to determine the orientation of the aerodynamic forces and their effect on the movement of the helicopter. But unfortunately, in the case of turbulence method does not work. Another technic is also given. It does not require all the blades to be in the same plane. 
It can be used to study the flapping motion of each blade in rotational coordinates [6] and consider the Coriolis force. The authors provide two illustrations of the blade models: the equivalent articulated rotor and the equivalent method for rigid blade flapping. However, for accurate studies of the helicopter dynamics blade model cannot be used if it is rigid in the flapping motion. It is necessary to consider the elastic model constructed using FEM.

The authors in [7] carry out a study of the helicopter in order to optimize the power and loads. They used the rotorcraft comprehensive aeromechanics analysis CAMRAD II [8] (a finite element code for a comprehensive analysis of rotorcraft). A quarter chord blade model in the form of a cantilever Bernoulli-Euler beam was considered to calculate the actuation power. The actuator was applied as a perpendicular force at the trailing edge, the preload was carried out by adding a spring to the model, and the aerodynamic force was considered by adding a distributed load. Each blade consisted of one rigid and seven elastic beam segments along the span of the blade. The blade aerodynamics were calculated with the help of the lifting-line theory which is based on two-dimensional profile characteristics at discrete radial stations. The airfoil polars for the NACA 23012 baseline and for curved profile shapes were calculated using CFD modeling based on RANS [9].

\section{Application of the Craig-Bampton method to problems with the resonance phenomenon}

The MSC Adams software uses the Craig-Bampton method with the help of approximation.

The examined case belongs to the high-frequency rotation class of problems. When the main rotor blade is accelerating, the external oscillation frequency at a certain moment approaches its natural frequency, which leads to such a phenomenon as resonance. As a result, the problem of convergence of the numerical solution arises. The blades bend as shown in figure 1 (a).

As the blade speed increases, the first natural frequency also increases, and its growth is accompanied by the strengthening effect of the blade. The described problem arises because the calculated eigenvalues and modes by the Craig-Bampton method do not consider this strengthening effect and remain unchanged when the blade speed is increased.

This problem has been solved by taking into account the preloaded state before the blade rotation dynamics calculation. Using boundary conditions, a load equivalent to the centrifugal forces was applied as the rotor rotates at operating frequency. As a result, the blade deformations correspond to a real representation. Blade deformations in the simulation without preloading are shown in figure 1 (a). Blade deformations in the simulation with preloading are shown in figure 1 (b). 


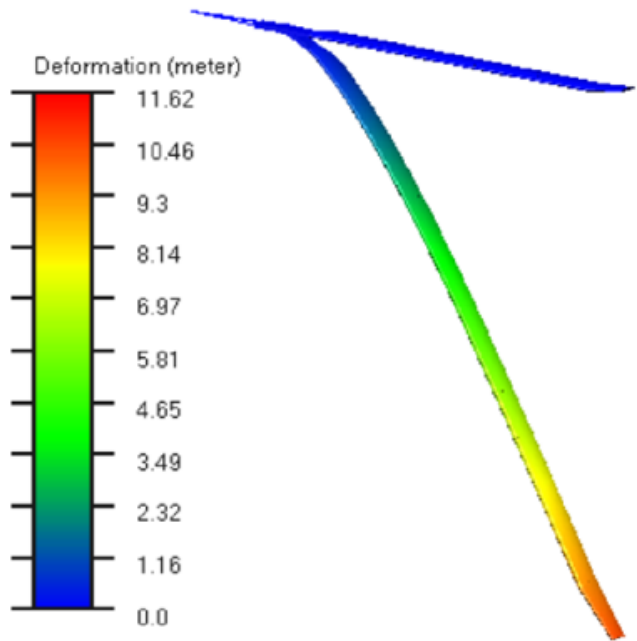

a)
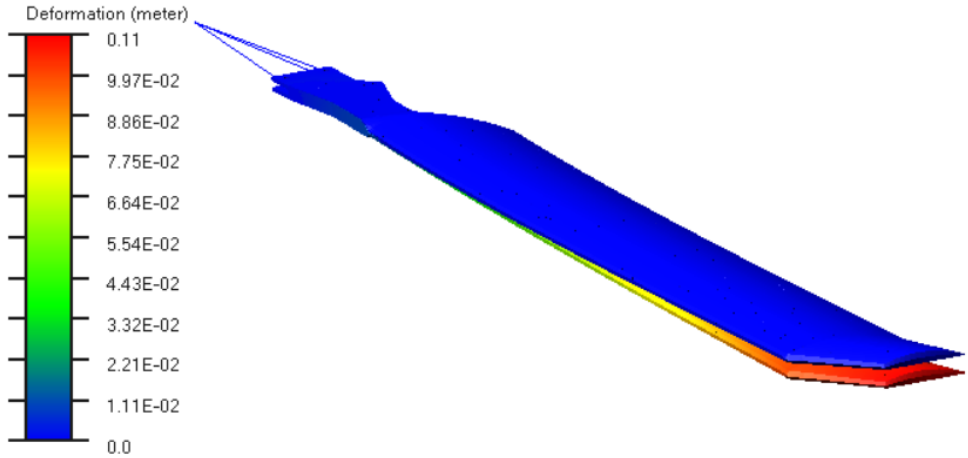

b)

Fig. 1. The deformed blade in comparison with the static position for the case of modeling a) without preloading, b) with preloading.

This method of solution is considered possible since all the calculated cases are performed at the same rotational operating frequency. In the future change is also not assumed. In the presence of a small coning angle, the model without preloading was observed to lose stability, while the pre-loaded model remained stable. It was shown that, depending on the preload, it was possible to pass the resonance phenomenon. Since then this method is applicable for such simulations and analyses.

Tables 1-3 and figures 2-8 show the results of the numerical solution. The following are the modes of flexural vibrations in two perpendicular directions and the modes of torsional vibrations. The corresponding eigenvalues of oscillations are also presented. 


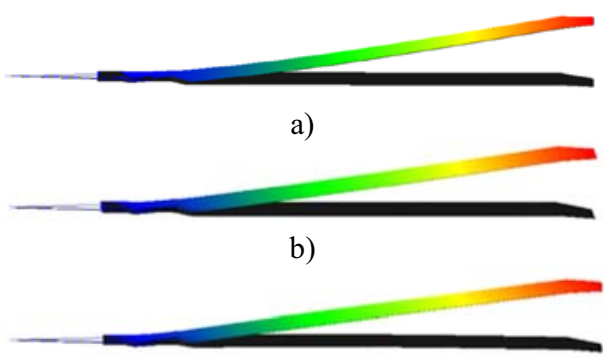

c)

Fig. 2. The first mode of flexural vibrations in vertical direction a) without preloading, b) with preloading $(5 \mathrm{~Hz}), \mathrm{c})$ with preloading $(10 \mathrm{~Hz})$.

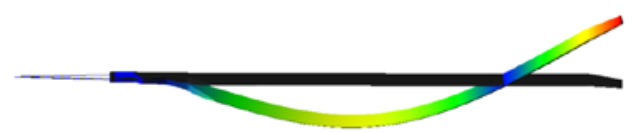

a)

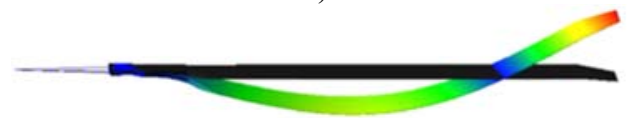

b)

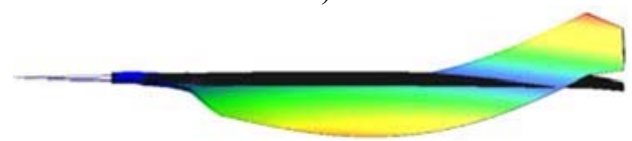

c)

Fig. 3. The second mode of flexural vibrations in vertical direction a) without preloading, b) with preloading $(5 \mathrm{~Hz})$, c) with preloading $(10 \mathrm{~Hz})$.

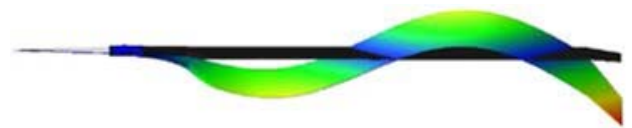

a)

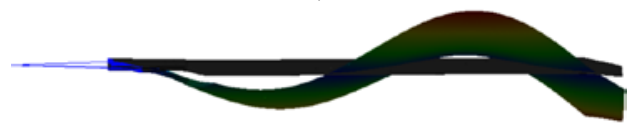

b)

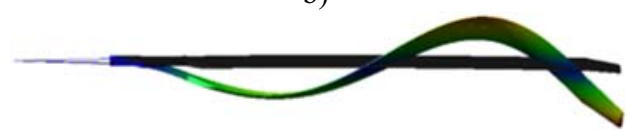

c)

Fig. 4. The third mode of flexural vibrations in vertical direction a) without preloading, b) with preloading $(5 \mathrm{~Hz}), \mathrm{c})$ with preloading $(10 \mathrm{~Hz})$. 


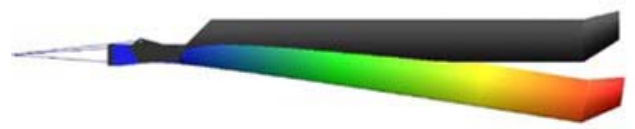

a)

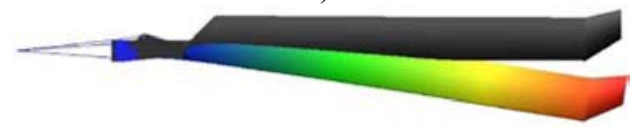

b)

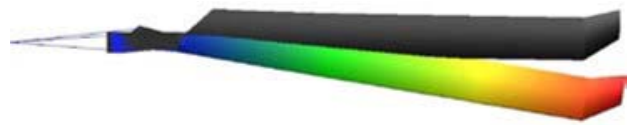

c)

Fig. 5. The first mode of flexural vibrations in horizontal direction a) without preloading, b) with preloading $(5 \mathrm{~Hz}), \mathrm{c})$ with preloading $(10 \mathrm{~Hz})$.

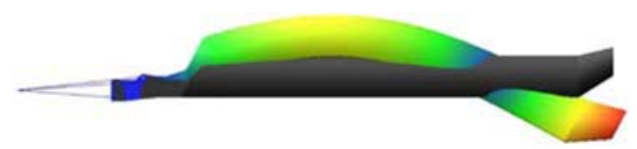

a)

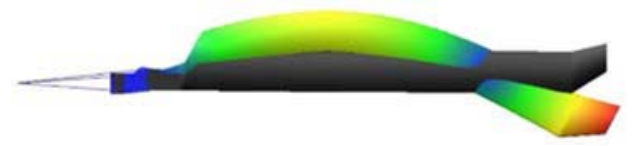

b)

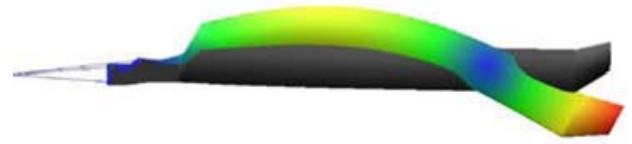

c)

Fig. 6. The second mode of flexural vibrations in horizontal direction a) without preloading, b) with preloading $(5 \mathrm{~Hz}), \mathrm{c})$ with preloading $(10 \mathrm{~Hz})$.

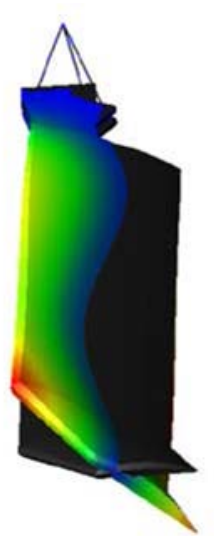

a)

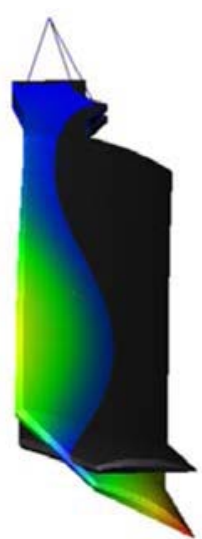

b)

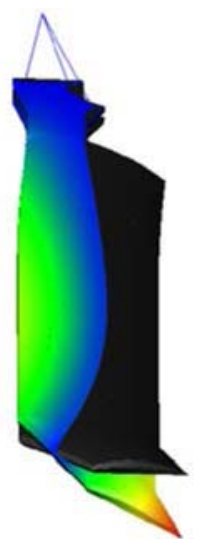

c)

Fig. 7. The first mode of torsional vibrations a) without preloading, b) with preloading $(5 \mathrm{~Hz})$, c) with preloading $(10 \mathrm{~Hz})$. 


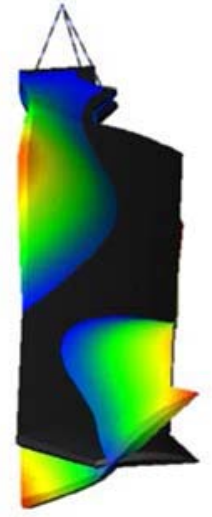

a)

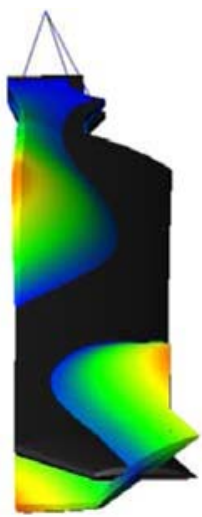

b)

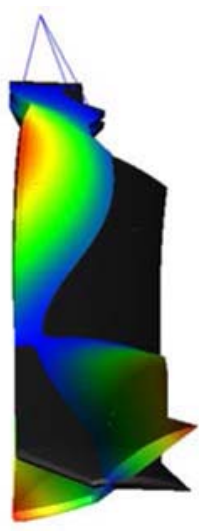

c)

Fig. 8. The second mode of torsional vibrations a) without preloading, b) with preloading (5 Hz), c) with preloading $(10 \mathrm{~Hz})$.

Table 1. Eigenvalues of flexural vibrations in vertical direction.

\begin{tabular}{|c|c|c|c|}
\hline \multirow{2}{*}{ № } & \multicolumn{3}{|c|}{ Frequency, Hz } \\
\cline { 2 - 4 } & Without preload & With preload (5 Hz) & With preload (10 Hz) \\
\hline 1 & 1.2 & 6.2 & 11.9 \\
\hline 2 & 8.4 & 16.8 & 29.1 \\
\hline 3 & 23.8 & 35.5 & 53.5 \\
\hline
\end{tabular}

Table 2. Eigenvalues of flexural vibrations in horizontal direction.

\begin{tabular}{|c|c|c|c|}
\hline \multirow{2}{*}{ № } & \multicolumn{3}{|c|}{ Frequency, Hz } \\
\cline { 2 - 4 } & Without preload & With preload (5 Hz) & With preload (10 Hz) \\
\hline 1 & 4.5 & 7.6 & 12.8 \\
\hline 2 & 44.7 & 47.0 & 53.0 \\
\hline
\end{tabular}

Table 3. Eigenvalues of torsional vibrations.

\begin{tabular}{|c|c|c|c|}
\hline \multirow{2}{*}{ № } & \multicolumn{3}{|c|}{ Frequency, Hz } \\
\cline { 2 - 4 } & Without preload & With preload (5 Hz) & With preload (10 Hz) \\
\hline 1 & 29.9 & 29.0 & 32.0 \\
\hline 2 & 95.5 & 98.1 & 101.7 \\
\hline
\end{tabular}

Based on the tables 1-3 it can be concluded that the method works great for the studying vibrations. When the blade rotation speed increases, the eigenvalues of flexural vibrations in vertical direction increase too. For vibrations in the horizontal plane, there is also an increase in frequencies, but not so significant. The eigenvalues of torsional vibrations do not change much from the magnitude of the centrifugal force, but this is quite expected.

\section{Conclusion}

In this paper, it is shown that the Craig-Bampton method makes it possible to apply the analytically or numerically obtained aerodynamic loads to the blade nodes and investigate the dynamics considering loads and deformations.

Summing up, in blade rotation it is necessary to take into account the inertial relationship between large body movements and elastic deformations, which allows us to conclude that the Craig-Bampton method is applicable to the rotation of the blades. At the same time, it is necessary to consider the practical problems that may arise in using general equations of motion. The most significant problems are: the presence of zero mass-inertia characteristics 
by some DOF in some practical cases and the incorrect description of the entire body behavior.

For comfortable usage of the Craig-Bampton method for a class of problems with highfrequency airscrew rotations, it is possible to use the MSC Adams software package. In order to minimize these practical troubles, the preloaded state should be considered to avoid the appearance of negative consequences [10-14].

\section{References}

1. V.V. Yotov, M. Remedia, G.S. Aglietti, G. Richardson, ActaAstronautica 178, 556-570 (2021)

2. T. Xian Bin, Z. Jun Dong, Polish Maritime Research 25, 30-34 (2018)

3. K.-L. Nguyen, Q.-T. Tran, M.-A. Andrianoely, L. Manin, S. Baguet, R. Dufour, M. Mahjoub, S. Menand, International Journal of Mechanical Sciences 166, 105225 (2020)

4. V.G. Boikov, I.V. Gaganov, F.F. Rafaelyevich, A.A. Yudakov, Chebyshevskii Sbornik 18(3), 131-153 (2017)

5. R. Chen, Y. Yuan, D. Thomson, Progress in Aerospace Sciences 120, 100681 (2021)

6. J.J. Howlett, UH-60A Black Hawk Engineering Simulation Program, Volume 1: Mathematical Model, NASA-CR-166309 (1981)

7. D. Komp, S. Kumar, M. Hajek, J. Rauleder, Aerospace Science and Technology 108, $106311(2021)$

8. W. Johnson, Technology drivers in the development of CAMRAD II, American Helicopter Society Aeromechanics Specialists Conference (1994)

9. A. Abdelmoula, J. Rauleder, Aerodynamic performance of morphed camber rotor airfoils, AIAA Scitech (2019)

10. A.D. Karpov, A.A. Zhilenkov, D. Lisitsa, IEEE Conference of Russian Young Researchers in Electrical and Electronic Engineering (EIConRus) 887 (2017) doi: 10.1109/EIConRus.2017.7910697

11. A.V. Ivanov, A.A. Zhilenkov, IEEE Conference of Russian Young Researchers in Electrical and Electronic Engineering (EIConRus) $\mathbf{8 8 2}$ (2018) doi: 10.1109/EIConRus.2018.8317229

12. S. Sokolov, A. Zhilenkov, S. Chernyi, A. Nyrkov, D. Mamunts, Symmetry 11, 236 (2019) https://doi.org/10.3390/sym11020236

13. A.P. Nyrkov, A.A. Zhilenkov, V.V. Korotkov, S.S. Sokolov, S.G. Chernyi, J. Phys.: Conf. Ser. 803, 012108 (2017) doi:10.1088/1742-6596/803/1/012108

14. A. A. Zhilenkov, D. Denk, IEEE Conference of Russian Young Researchers in Electrical and Electronic Engineering (EIConRus) 1104 (2017) doi: 10.1109/EIConRus.2017.7910748 